\title{
Acanthamoeba Keratitis Associated with Scleral Contact Lens Use in a Keratoconus Patient
}

\author{
Dehmet Ozgur Cubuk, ${ }^{1}$ () Funda Dogruman Al, ${ }^{3}$ (D) Selma Usluca, ${ }^{4}$ (D) Kamil Bilgihan ${ }^{2}$ \\ ${ }^{1}$ Department of Ophthalmology, Istanbul Training and Research Hospital, Istanbul, Turkey \\ ${ }^{2}$ Department of Ophthalmology, Gazi University Faculty of Medicine, Ankara, Turkey \\ ${ }^{3}$ Department of Microbiology, Gazi University Faculty of Medicine, Ankara, Turkey \\ ${ }^{4}$ Department of Parasitology, National Public Health Institution, Ankara, Turkey
}

\begin{abstract}
To the best of our knowledge, this is the second reported case of Acanthamoeba keratitis (AK) as a result of scleral lens use and the first case of AK associated with Maxim scleral lens use (Accu-Lens, Inc., Lakewood, CO, USA). A 22-year-old male scleral lens user presented at the department of ophthalmology at Gazi University Hospital complaining of painful corneal opacities and erosion in the cornea of right eye. A real-time polymerase chain reaction assay (Primerdesign, Southampton, UK) was performed, and Acanthamoeba spp. DNA was amplified on the corneal specimen. A topical antimicrobial treatment was prescribed, and the symptoms had improved significantly at the 2-week follow-up. Contact lens wearers always run the risk of developing AK, even with gas-permeable scleral contact lenses. Therefore, AK must be considered as an important differential diagnosis in patients who use scleral contact lenses.
\end{abstract}

Keywords: Acanthamoeba keratitis, scleral contact lens.

\section{Introduction}

Acanthamoeba is an uncommon but serious cause of corneal infection. Even though the protozoon is ubiquitous in our environment, Acanthamoeba keratitis (AK) is relatively rare (I). Corneal infection caused by Acanthamoeba was first described in the early 1970s $(2,3)$. Soft contact lens use is an important and well-known risk factor for AK; as many as $70 \%$ of reported cases are associated with contact lens use (4-6). Noncompliance with contact lens hygiene protocols and swimming while wearing contact lenses are important predisposing factors for AK (4-6). Although soft contact lens use is a well-known risk factor for $A K$, there is only I reported case of $A K$ associated with scleral lens use (7). To the best of our knowledge, the current case is the second known case of $A K$ in a scleral lens user and the first case of $A K$ in a Maxim scleral lens user (Accu-Lens, Inc., Lakewood, CO, USA). A topical treatment regimen led to a successful outcome.

\section{Case Report}

A 22-year-old man presented at the department of ophthalmology at Gazi University Hospital complaining of painful corneal opacities and erosion in his right eye beginning 3 days prior. The patient reported a history of bilateral keratoconus and Maxim scleral lens use for 2 years to improve his visual acuity. He also reported poor compliance with contact lens hygiene protocols. He complained of impaired visual acuity (0.05 with Snellen lines) and eye pain, and de-

Address for correspondence: Mehmet Ozgur Cubuk, MD. Istanbul Egitim ve Arastirma Hastanesi, Goz Hastaliklari Anabilim Dali, Istanbul, Turkey

Phone: +90 5057901038 E-mail: mehmetozgurcubuk@yahoo.com

Submitted Date: October 14, 2019 Accepted Date: December 18, 2019 Available Online Date: February 17, 2020

${ }^{\circ}$ Copyright 2020 by Beyoglu Eye Training and Research Hospital - Available online at www.beyoglueye.com OPEN ACCESS This work is licensed under a Creative Commons Attribution-NonCommercial 4.0 International License. 
scribed the condition as like sinking underwater. On the first day he presented at the clinic, a slit-lamp examination revealed stromal and perineural infiltrates in the right eye (Fig. IA). Topical aztreonam (Azactam; Bristol-Myers Squibb Co., New York, NY, USA) was applied 7 times per day. After 2 days, there was no improvement in his symptoms and no improvement was observed upon slit-lamp examination. A corneal epithelial debridement was performed to improve drug penetration and eliminate any surface organisms. A real-time polymerase chain reaction (RTPCR) assay (Primerdesign, Southampton, UK) was used on a corneal specimen to investigate the diagnosis of AK. A culture was performed to examine for bacteria and fungi, and Giemsa stain was added for a histological diagnosis of the presence of trophozoites or cysts. Anterior segment optical coherence tomography revealed a highly reflective line-match with keratoneuritis (Fig. IB) (8). Topical chlorhexidine 7 times per day (0.02\%), voriconazole 7 times per day $(10 \mathrm{mg} / \mathrm{mL}$, Vfend; Pfizer, Inc., NY, NY, USA), propamidine 4 times per day (propamidine isetionate $0.1 \%$, Brolene eye drops; Sanofi, SA, Paris, France), and oral itraconazole once per day (200 mg, Funit; Nobel llac, Umraniye, Turkey) were added. After 2 days, Acanthamoeba spp. DNA was amplified on a corneal specimen via RTPCR. Trophozoites of Acanthamoeba spp. were identified in a Giemsa stain. Culture tests were negative for fungi and bacteria
(Fig. ID). The patient's symptoms had improved significantly at a 2-week follow-up. A I-month follow-up slit-lamp examination showed improvement in the stromal and perineural infiltrates (Fig. ID). Topical aztreonam was terminated, and oral itraconazole was continued for 3 more weeks. Use of topical chlorhexidine 7 times per day, voriconazole 7 times per day, and propamidine 4 times per day was maintained for 3 months.

\section{Discussion}

Gas-permeable scleral contact lenses (GP-ScCL) have been shown to play a significant role in the treatment of multiple types of ocular surface diseases and corneal ectasias (9-II). Nonetheless, the risk of acquiring microbial keratitis is always present, even with GP-ScCL. Corneas with ocular surface disease are particularly prone to keratitis due to the deficiency of the natural defenses provided by the lids, tears, epithelium, and basal lamina (I2). An intact tear film is crucial to avoiding microbial keratitis. Soft contact lenses are associated with a thin post-lens tear film and a smaller volume of post-lens tear exchange when compared with gas-permeable lenses; GP-ScCL ensure a thick post-lens tear film (I3).

Previous studies have reported low rates of infectious keratitis with GP-ScCL use. A cohort study that included 33 patients using GP-ScCL for chronic graft versus host disease
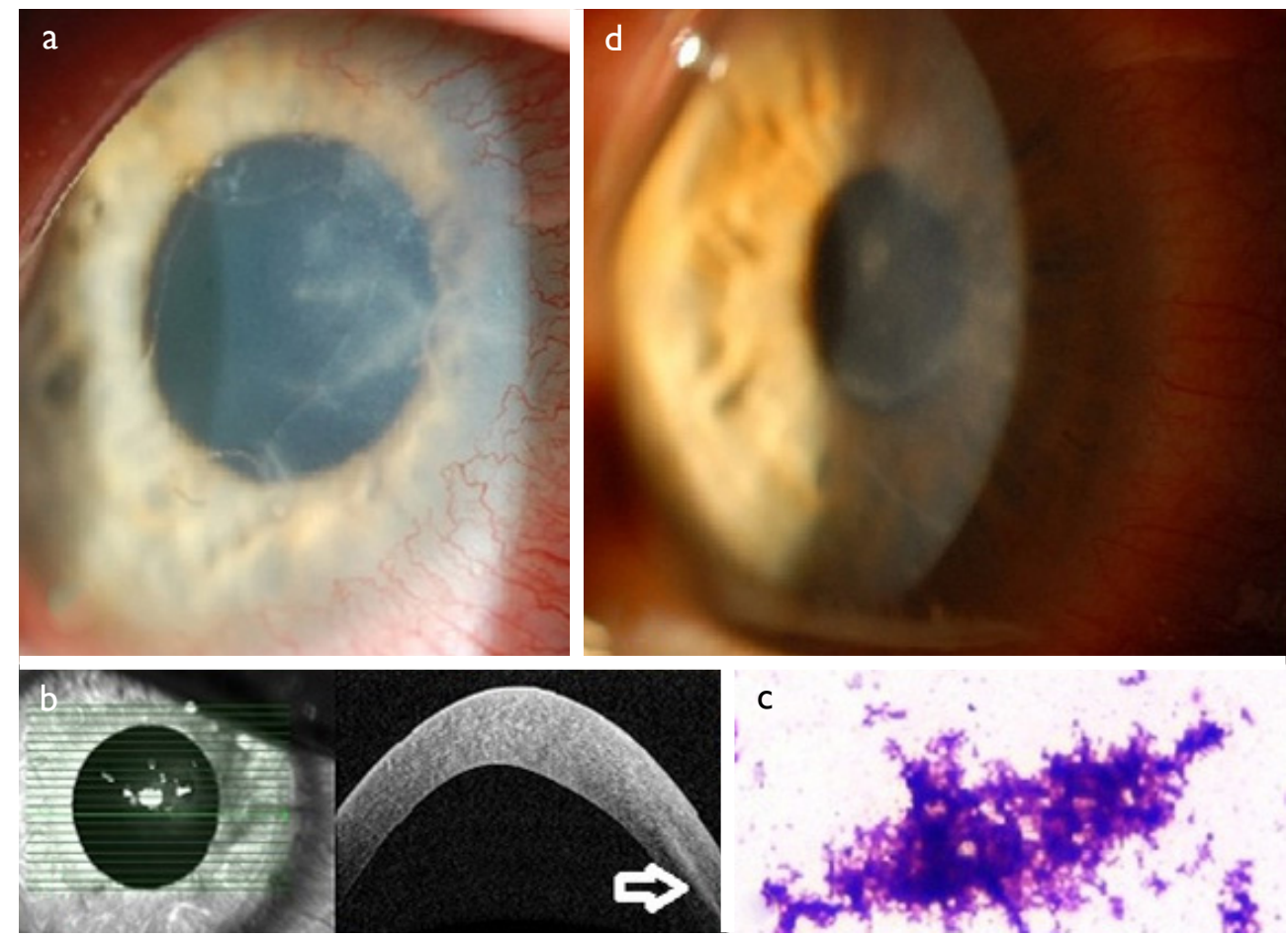

Figure I. (a) Slit-lamp examination revealed stromal and perineural infiltrates in the right eye; (b) Highly reflective line-matching with keratoneuritis was observed with anterior segment optic coherence tomography; (c) Acanthamoeba species trophozoites with spine-like pseudopods (Giemsa stain, 100x); (d) Slit-lamp examination revealed improvement in the stromal and perineural infiltrates I month after the patient presented at the clinic. 
(cGvHD) demonstrated no infectious keratitis (I4). In contrast, the same authors found 4 cases of microbial keratitis among 13 patients using fenestrated scleral lenses for persistent epithelial keratitis (I5). In addition, a study on corneal ectasia patients wearing GP-ScCL found no instance of corneal infection (16).

To the best of our knowledge, there has only been I reported case of $A K$ in a sclera lens user. The patient had used scleral lenses for 5 years to improve symptoms of cGvHD and dry eye syndrome (DES). The patient had also been treated with deep anterior lamellar keratoplasty (7). We present the second case of AK in a scleral lens user and the first case in a Maxim scleral lens user, with a successful result using topical treatment.

Acanthamoeba expresses a mannose-binding protein (MBP) that enables them to attach to the corneal surface, after which they degrade the epithelial basement membrane and stroma via proteinases (17). Human tears contain antiMBP immunoglobulin $A$, which protects the eye against AK (18). The previously mentioned case report indicated that decreased tear production caused by CGvHD was an important predisposing factor for AK (7). Our patient had no history of cGvHD or DES; therefore, this was not a predisposing factor in this case.

Animal trials have shown that chronic use of systemic steroids can enhance the pathogenicity of Acanthamoeba in the eye (19). Farhat et al. (7) thought that the history of chronic systemic steroid use in their patient made AK unresponsive to topical therapy. This can explain our successful result with topical treatment in our patient who had no history of systemic steroid use.

Contact lens contamination with tap water is a well-known risk factor for AK (4-6). Appropriate contact lens care is crucial to inhibit contact with Acanthamoeba in tap water. Our patient reported poor compliance with contact lens hygiene protocols and he also reported that he did not use a hydrogen peroxide solution for lens cleaning and storage. Therefore, we think that poor compliance with contact lens hygiene protocols was the main risk factor for our patient.

There are no drugs specifically for AK. Although trophozoites are sensitive to topical treatment, cysts are resistant. Medical therapy can include $1.0 \%$ povidone-iodine, $0.05 \%$ benzalkonium chloride, polyhexamethylene biguanide $0.02 \%$ (PHMB), chlorhexidine $0.02 \%$, hexamidine $0.1 \%$, propamidine isethionate $0.1 \%, 5.0 \%$ natamycin, and $1.0 \%$ voriconazole $(20,2 \mathrm{I})$. Sunada et al. (2I) reported that natamycin and povidone-iodine had cysti-static (or cystcidal) effects; however, PHMB and propamidine did not. They also found that none of the Acanthamoeba strains was susceptible to voriconazole, the susceptibility to PHMB and chlorhexidine may be time-dependent, and the susceptibility to propami- dine may be concentration dependent (2I). However, voriconazole has been demonstrated to be an effective agent in some studies $(22,23)$. Corneal epithelial debridement and keratoplasty are alternatives for refractory cases. The treatment can improve drug penetration and eliminate any surface organisms (4). Topical chlorhexidine 7 times a day, voriconazole 7 times a day, and propamidine 4 times a day was used in the present case after the diagnosis. We had successful results after 2 weeks of topical treatment and topical therapy was continued for 3 months.

\section{Conclusion}

In conclusion, though studies have indicated that the rate of infectious keratitis with GP-ScCL use is low, the risk of acquiring $A K$ is always present. $A K$ is an important differential diagnosis in patients who use scleral lenses.

\section{Disclosures}

Informed consent: Written informed consent was obtained from the patient for the publication of the case report and the accompanying images.

Peer-review: Externally peer-reviewed.

Conflict of Interest: None declared.

Authorship Contributions: Involved in design and conduct of the study (MOC, KB); preparation and review of the study (MOC, $K B$ ); data collection (MOC, FDA, SU, KB).

\section{References}

I. Anger C, Lally JM. Acanthamoeba: A review of its potential to cause keratitis, current lens solution disinfection standards and methodologies and strategies to reduce patient risk. Eye Contact Lens 2008;34:247-53.

2. Visvesvara GS. Free-living pathogenic amoeba. In: Lennette EH, Balows A, Hausler WJ Jr, editors. Manual of Clinical Microbiology. 3rd ed. Washington, DC: American Society of Microbiology 1980. p. 704-8.

3. Naginton J, Watson PG, Playfair TJ, McGill J, Jones BR, Steele AD. Amoebic infection of the eye. Lancet 1974;2:1537-40.

4. Dart JK, Saw VP, Kilvington S. Acanthamoeba keratitis: Diagnosis and treatment update 2009. Am J Ophthalmol 2009;148:48799.e2.

5. Radford CF, Bacon AS, Dart JK, Minassian DC. Risk factors for acanthamoeba keratitis in contact lens users: A case-control study. BMJ 1995;310:1567-70.

6. Schaumberg DA, Snow KK, Dana MR. The epidemic of Acanthamoebakeratitis:Where do we stand? Cornea 1998; 17:3-10.

7. Farhat B, Sutphin JE. Deep anterior lamellar keratoplasty for acanthamoeba keratitis complicating the use of Boston scleral lens. Eye Contact Lens 2014;40:e5-7.

8. Yamazaki N, Kobayashi A, Yokogawa H, Ishibashi Y, Oikawa Y, 
Tokoro M, et al. In Vivo Imaging of Radial Keratoneuritis in $\mathrm{Pa}$ tients with Acanthamoeba keratitis by Anterior-Segment Optical Coherence Tomography. Ophthalmology 2014;121:2153-8.

9. Pullum KW, Whiting MA, Buckley RJ. Scleral contact lenses: The expanding role. Cornea. 2005;24:269-77.

10. Segal O, Barkana Y, Hourovitz D, Behrman S, Kamun Y, Avni I, et al. Scleral contact lenses may help where other modalities fail. Cornea 2003;22:308-10.

I I. Romero-Rangel T, Stavrou P, Cotter J, Rosenthal P, Baltatzis S, Foster CS. Gas-permeable scleral contact lens therapy in ocular surface disease. Am J Ophthalmol 2000;130:25-32.

12. Fleiszig SM, Evans DJ. Pathogenesis of contact lens-associated microbial keratitis. Optom Vis Sci 2010;87:225-32.

13. Zimmerman AB, Marks A. Microbial keratitis secondary to unintended poor compliance with scleral gas-permeable contact lenses. Eye Contact Lens 20I4;40:el-4.

14. Jacobs DS, Rosenthal P. Boston scleral lens prosthetic device for treatment of severe dry eye in chronic graft-versus-host disease. Cornea 2007;26:1 195-9.

15. Rosenthal P, Cotter JM, Baum J. Treatment of persistent corneal epithelial defect with extended wear of a fluid-ventilated gas-permeable scleral contact lens. Am J Ophthalmol 2000; 130:33-4I.

16. Baran I, Bradley JA, Alipour F, Rosenthal P, Le HG, Jacobs DS. PROSE treatment of corneal ectasia. Cont Lens Anterior Eye
2012;35:222-7.

17. Panjwani N. Pathogenesis of Acanthamoeba keratitis. Ocul Surf 2010;8:70-9.

18. Cao Z, Saravanan C, Goldstein MH, Wu HK, Pasricha G, Sharma S, et al. Effect of human tears on Acanthamoeba- induced cytopathic effect. Arch Ophthalmol 2008;126:348-52.

19. McClellan K, Howard K, Niederkorn JY, Alizadeh H. Effect of steroids on Acanthamoeba cysts and trophozoites. Invest Ophthalmol Vis Sci 2001;42:2885-93.

20. Por YM, Mehta JS, Chua JL, Koh TH, Khor WB, Fong AC, et al. Acanthamoeba keratitis associated with contact lens wear in Singapore. Am J Ophthalmol 2009;|48:7-12.e2.

2I. Sunada A, Kimura K, Nishi I, Toyokawa M, Ueda A, Sakata T, et al. In Vitro Evaluations of Topical Agents to Treat Acanthamoeba Keratitis. Ophthalmology 20|4; I21:2059-65.

22. Cabello-Vílchez AM, Martín-Navarro CM, López-Arencibia A, Reyes-Batlle M, Sifaoui I, Valladares B, et all. Voriconazole as a first-line treatment against potentially pathogenic Acanthamoeba strains from Peru. Parasitol Res 2014; I 13:755-9.

23. lovieno A, Miller D, Ledee DR, Alfonso EC. Cysticidal activity of antifungals against different genotypes of Acanthamoeba Antimicrob Agents Chemother. Antimicrob Agents Chemother 20|4;58:5626-8. 